\title{
Actividad física en niños españoles. Factores asociados y evolución 2003-2006
}

\author{
C. Casado Pérez ${ }^{\mathrm{a}}$, N. Alonso Fernández ${ }^{\mathrm{b}}$, V. Hernández Barrerac ${ }^{\mathrm{c}}$ R. Jiménez García ${ }^{\mathrm{d}}$ \\ ${ }^{a}$ Enfermera. CS Baviera, Servicio Madrileño de Salud, Área 2. Madrid. España. \\ bLicenciada en Biología. Técnico de Laboratorio. Departamento de Ciencias de la Salud I, \\ Universidad Rey Juan Carlos. Alcorcón, Madrid. España. \\ 'Estadístico. Departamento de Ciencias de la Salud I, Universidad Rey Juan Carlos. Alcorcón, Madrid. España. \\ ${ }^{`}$ Profesor. Doctor en Medicina. Departamento de Ciencias de la Salud I, \\ Universidad Rey Juan Carlos. Alcorcón, Madrid. España.
}

Rev Pediatr Aten Primaria. 2009;1 I:219-31
Carmen Casado Pérez, carmencp26@hotmail.com

Resumen

Introducción: el sedentarismo es uno de los principales problemas de la sociedad actual. Este estudio analiza los principales factores que inciden en los hábitos de actividad física en jóvenes españoles entre 6 y 15 años, y determina las principales tendencias de la actividad física en el tiempo libre entre el año 2003 y 2006.

Métodos: estudio descriptivo realizado con los datos de la Encuesta Nacional de Salud (ENS) de 2003 y 2006. El tamaño de la muestra es de 4.420 niños entre 6 y 15 años en el 2003 y de 5.771 en el 2006. Se realizó un primer análisis univariante, una regresión logística multivariante y finalmente calculamos la tendencia.

Resultados: el 78,3\% y 89,1\% de esta población eran niños activos en el 2003 y 2006 respectivamente. El porcentaje de niños activos era superior al de niñas para todas las categorías de edad, excepto para el rango de edad de 6-7 años del 2003. Los niños con una buena salud (valorada por sus padres) eran más activos que los que no la tenían. Los jóvenes cuyos padres tienen niveles de educación altos practican más actividad física que los de padres con estudios inferiores.

Conclusiones: la juventud realiza más ejercicio en su tiempo libre conforme aumenta su edad. Las estrategias dirigidas a la promoción de la actividad física en esta población deben tener en cuenta las variables edad, sexo, percepción de la salud y nivel de estudios del cabeza de familia principalmente.

Palabras clave: Actividad física, Actividad en el tiempo libre, Hábitos alimenticios, Ingresos, Obesidad, Educación, Estilo de vida.

Abstract

Introduction: sedentary lifestyle is one of the main problems in present-day society. This study analyzes the main factors that have an influence on the physical activity habits among

Los autores declaran no presentar conflictos de intereses en relación con la preparación y publicación de este artículo. 
Spanish young people aged 6 to 15 and determines its main trends during leisure time from 2003 to 2006.

Methods: this is a cross-sectional study. The sample size is 4,420 children aged 6 to 15 in 2003 and 5,771 in 2006. A first univariate analysis is carried out and then, with the resulting significant variables, a multivariant logistic regression is made. Finally, the trend is determined.

Results: seventy-eight point three percent and the $89.1 \%$ of these children were active in 2003 and 2006 respectively. The percentage of active boys was higher than that of girls for all age categories, except for children aged 6 to 7 in 2003. Children with a good health (considered by their parents) were more active than those without it. Those young people whose parents have high educational levels, practice more physical activity than those whose parents have lower studies.

Conclusions: youth practise more exercise during their leisure time ultimately. The strategies directed to promote physical activity in children must take into account characteristics of age, sex, health perception and educational level of the head of the household.

Key words: Physical activity, Leisure activity, Food habits, Income, Obesity, Education, Lifestyle.

\section{Introducción}

La actividad física es un elemento esencial en el establecimiento de una situación ideal de salud ${ }^{1}$. Si a cualquier edad el ejercicio físico es recomendable, todavía lo será más en el niño y el adolescente, ya que puede considerarse como un elemento básico para el desarrollo1.

Muchos años de investigación han demostrado que existen efectos positivos en la realización de actividad física y deporte en la salud física y en el bienestar psicológico ${ }^{2}$. Existen evidencias que sugieren que esta actividad durante la adolescencia tiene beneficios para la salud actual y futura ${ }^{2}$.

La actividad física reduce el riesgo de padecer enfermedades coronarias y cardiovasculares; cáncer de colon; diabetes mellitus no insulinodependiente ${ }^{3,4}$; tensión arterial alta y obesidad, ayudando a controlar el sobrepeso ${ }^{5}$; fortalece los huesos aumentando la densidad ósea; fortalece los músculos ${ }^{3,6}$; y mejora la salud psicológica, aumentando los niveles de autoestima y disminuyendo los niveles de ansiedad, depresión y estrés ${ }^{7}$. Fomenta la sociabilidad, la integración social y en niños, además, mejora la maduración del sistema nervioso motor, aumenta las destrezas motrices y mejora el rendimiento escolar.

También contribuye, junto a la dieta, a la regulación del peso corporal y a la lucha contra la obesidad y, por tanto, a la prevención de esta enfermedad ${ }^{1}$. La prevalencia de la obesidad infantil ha subido a nivel mundial durante las pasadas déca- 
das 9 . En España, según la Encuesta Nacional de Salud (ENS) de 2003, el 8,5\% de los niños entre 2 y 17 años tienen obesidad, mientras que el $18,2 \%$ presenta sobrepeso, lo cual supone el $7 \%$ del gasto sanitario total ${ }^{10}$. Con estos datos, la población infantil de nuestro país presenta una de las cifras más altas ${ }^{10}$.

Sin duda, la actividad física aporta beneficios de salud físicos, mentales y sociales, y a pesar de todas las ventajas, en nuestra sociedad ${ }^{11}$, el estilo de vida de los niños está determinado por el sedentarismo, el estrés, el consumo de tabaco, alcohol y drogas, la dieta y el mayor número de horas de televisión (TV ${ }^{12}$. Hay presentes varios factores en las vidas de los jóvenes que dificultan la práctica deportiva, algunos de estos son el tipo de alimentación, la percepción del propio estado de salud, el descanso diario, la educación recibida, el estatus social, etc.

El estatus social es un factor clave para la salud, ya que se cree que existe un vínculo entre la actividad física en adolescentes y el estatus social de la familia. Se piensa que los adolescentes que provienen de familias con altos ingresos ${ }^{13}$ y padres con altos niveles de educación ${ }^{14-16}$ realizan una intensa actividad física.

También existe relación entre el deporte y el estilo de alimentación. Así, cuanto mayor es la percepción de competencia deportiva, mayor es la práctica de deporte y mayor el consumo de alimentos sanos ${ }^{17}$.

Y con respecto a la relación con la autoestima, los que sí participan en deportes y actividades físicas piensan que están mejorando su forma física y tienen una mejor autopercepción de su salud².

El objetivo principal del presente estudio es determinar los principales factores que inciden en los hábitos de actividad física en los jóvenes de entre 6 y 15 años durante los años 2003 y 2006; y con ello determinar las principales tendencias de la actividad física en el tiempo libre de esta población según variables independientes.

\section{Material y métodos}

Se realiza un estudio transversal acerca de la prevalencia de actividad física.

La población estudio está compuesta por niños españoles de 6 a 15 años de edad incluidos en las ENS (realizada por el Ministerio de Sanidad y Consumo) de 2003 y 2006. La muestra procede de secciones censales utilizadas en el Padrón Municipal de 2002 y de 2005. El tamaño de la muestra es de 4.420 niños/as en el año 2003 y de 5.771 en el 2006 (un total de 10.191 niños/as) entrevistados mediante el Cuestionario de Menores de la ENS 2003 y 2006. Se realizó un mues- 
treo polietápico estratificado donde las unidades de primera etapa son las secciones censales y las unidades de segunda etapa son las viviendas familiares principales. En todos los hogares donde había personas de 0 a 15 años, se seleccionó aleatoriamente a un único menor ${ }^{18,21}$.

La recogida de la información se hizo a lo largo de un año, (desde abril de 2003 a marzo de 2004 y desde junio de 2006 hasta junio de 2007), por un entrevistador preparado para esta tarea. Este realizó una entrevista personal, complementada cuando era necesario, mediante entrevista telefónica, al padre o madre del menor, y en su defecto al tutor o persona responsable de este ${ }^{18,21}$.

La variable de interés de este estudio es el ejercicio físico desarrollado en tiempo libre. Se define actividad física como cualquier movimiento corporal producido por los músculos esqueléticos que da como resultado un gasto calórico, para lo cual debe tener determinadas características de intensidad, duración y frecuencia. Según la frecuencia esta variable se clasifica en "no realiza actividad física alguna", o "realiza alguna actividad física o deportiva una o varias veces a la semana".

Las variables independientes están clasificadas en tres grupos:
1. Estado físico y mental del niño/a: en el que se incluyen edad, sexo, índice de masa corporal (IMC) y percepción de la salud. La edad se ha recodificado en 5 categorías de 2 años cada una. El IMC en bajo, normal y alto, calculado mediante peso y altura $\left(\mathrm{kg} / \mathrm{m}^{2}\right)$ según las recomendaciones de niños españoles por edad y sexo; considerándose adecuado para edades entre 6 y 8 años un IMC de 15,1 a 16; entre 9 y 10 años, de 16,1 a 17; entre 11 y 12 años, de 17,1 a 18; a los 13 años, de 18,1 a 19; y entre 14 a 15 años, de 19,1 a 20. La percepción que tienen los padres sobre el estado de salud de su hijo/a está clasificada en bueno, regular y malo.

2. Estilo de vida: comprende tipo de dieta y horas de descanso. El tipo de dieta está clasificado según se trate de una dieta sana o una inadecuada ${ }^{22}$. El número de horas de descanso, es decir, el número total de horas que se duerme al día habitualmente, incluidas las horas de la siesta, está organizado en deficiente y adecuado; estableciendo como adecuado: 12 horas para niños/as de 6 a 9 años; más de 9 horas para los que tengan entre 10 y 12 años; y de 8 a 9,5 horas para adolescentes o mayores de 13 años.

3. Sociodemografía: incluye tamaño del municipio, nivel de estudios terminados y el estatus social. El tamaño del mu- 
nicipio está clasificado en tres grupos: hasta 10.000 habitantes; de 10.001 a 50.000 habitantes; y de más de 50.001 habitantes (excepto capitales de provincia). El nivel de estudios terminados por el cabeza de familia se clasificó en tres grupos: estudios primarios y secundarios de primer ciclo o analfabetos; estudios secundarios de segundo ciclo y postsecundarios; y estudios universitarios. Por último, el estatus social esta dividido en cuatro: la I la forman altos cargos, titulaciones superiores, artistas y deportistas; la II son administrativos, de servicios personales y seguridad, autónomos y supervisores; la III son trabajadores manuales; y la IV, otras.

Respecto al análisis estadístico, primero se calculó y se comparó la proporción de sujetos que realizaban actividad física (prevalencia) según las variables de estudio para el año 2003 y 2006.

En segundo lugar, se utilizó el método estadístico del chi cuadrado para obtener comparaciones binarias de las proporciones y se fijó la significación estadística en un $\alpha<0,05$.

Luego, se realizó un análisis de regresión logística multivariante, utilizando como variable dependiente la actividad física, para determinar si existía asociación entre alguna de las variables independientes y dicha variable.
En cuarto lugar, para evaluar la tendencia general de las prevalencias de actividad física durante el período 2003-2006, se fusionaron las bases de datos de la ENS de ambos años, y se calculó mediante regresión logística multivariante la odds ratio (OR) ajustada de la actividad física en 2006 respecto a 2003. Todas las estimaciones se hicieron mediante la incorporación de diseños complejos de muestreo y factores de ponderación, usando las funciones "svy" (encuesta de comandos) del programa STATA $8.0^{\circledR}$, que nos ha permitido calcular las proporciones, los intervalos de confianza, chi cuadrado y regresión logística.

\section{Resultados}

La distribución de la actividad física en el tiempo libre según la edad y género se encuentra en la tabla I. Estos resultados revelan que el 78,3\% de la población en estudio del año 2003 realizaba actividad física en el tiempo libre, mientras que en el año 2006 fue de un 89,1\%. Por tanto, la población del 2006 es más activa que la del 2003. El porcentaje de niños activos era superior al de niñas activas para todas las categorías de edad, excepto para el rango de edad de 6-7 años del 2003.

El mayor porcentaje de niños activos lo encontramos en los rangos de edad entre los 8 y los 11, mientras que este por- 


\begin{tabular}{|c|c|c|c|c|c|}
\hline \multirow[t]{2}{*}{ Edad } & \multirow[t]{2}{*}{ Sexo } & \multicolumn{2}{|c|}{2003} & \multicolumn{2}{|r|}{2006} \\
\hline & & $\begin{array}{l}\mathrm{N} .{ }^{\circ} \text { de } \\
\text { sujetos }\end{array}$ & $\begin{array}{l}\text { Prevalencia de } \\
\text { ejercicio físico }\end{array}$ & $\begin{array}{l}\mathrm{N} .^{\circ} \text { de } \\
\text { sujetos }\end{array}$ & $\begin{array}{l}\text { Prevalencia de } \\
\text { ejercicio físico }\end{array}$ \\
\hline \multirow[t]{3}{*}{$6-7$} & Niño & 281 & $77,4 \%$ & 441 & $91,5 \%$ \\
\hline & Niña & 284 & $78,9 \%$ & 465 & $90,6 \%$ \\
\hline & Total & 565 & $78,1 \%$ & 906 & $91,1 \%$ \\
\hline \multirow{3}{*}{$\begin{array}{l}8-9 \\
*\end{array}$} & Niño & 322 & $88,2 \%$ & 467 & $94,3 \%$ \\
\hline & Niña & 252 & $73,7 \%$ & 478 & $91,4 \%$ \\
\hline & Total & 574 & $81,2 \%$ & 945 & $92,8 \%$ \\
\hline \multirow{3}{*}{$\begin{array}{l}10-11 \\
* / * *\end{array}$} & Niño & 401 & $87,2 \%$ & 553 & $94,5 \%$ \\
\hline & Niña & 327 & $77,1 \%$ & 475 & $89,3 \%$ \\
\hline & Total & 728 & $82,4 \%$ & 1.028 & $92,0 \%$ \\
\hline \multirow{3}{*}{$\begin{array}{l}12-13 \\
* / * *\end{array}$} & Niño & 478 & $86,6 \%$ & 571 & $89,6 \%$ \\
\hline & Niña & 356 & $73,0 \%$ & 519 & $84,1 \%$ \\
\hline & Total & 834 & $80,2 \%$ & 1.090 & $86,9 \%$ \\
\hline \multirow{3}{*}{$\begin{array}{l}14-15 \\
* / * *\end{array}$} & Niño & 435 & $81,9 \%$ & 620 & $89,9 \%$ \\
\hline & Niña & 327 & $61,1 \%$ & 493 & $78,3 \%$ \\
\hline & Total & 762 & $71,5 \%$ & 1.113 & $84,3 \%$ \\
\hline \multirow{3}{*}{$\begin{array}{l}\text { Total } \\
* / * *\end{array}$} & Niño & 1.917 & $84,4 \%$ & 2.652 & $91,8 \%$ \\
\hline & Niña & 1.546 & $71,9 \%$ & 2.430 & $86,3 \%$ \\
\hline & Total & 3.463 & $78,3 \%$ & 5.082 & $89,1 \%$ \\
\hline
\end{tabular}

${ }^{*}$ Asociación significativa 2003. * *Asociación significativa 2006.

Tabla II. Distribución de la actividad física en el tiempo libre según variables de estilo de vida

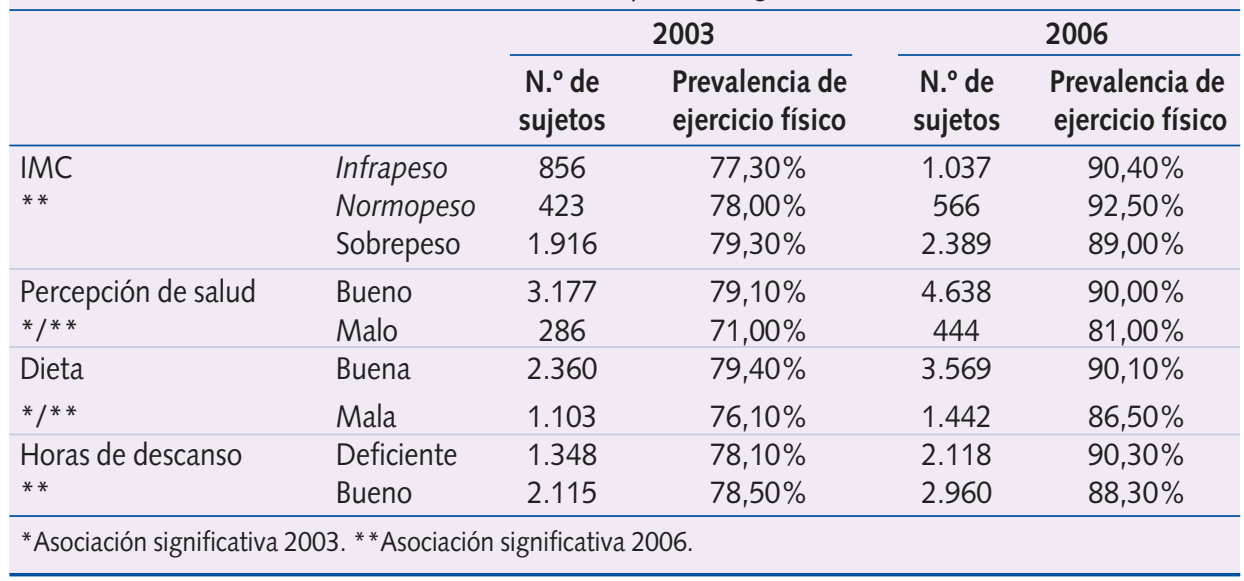


Tabla III. Distribución de la actividad física en el tiempo libre según variables sociodemográficas

\begin{tabular}{|c|c|c|c|c|c|}
\hline & \multicolumn{2}{|c|}{2003} & \multicolumn{2}{|r|}{2006} \\
\hline & & $\begin{array}{l}\text { N. }{ }^{\circ} \text { de } \\
\text { sujetos }\end{array}$ & $\begin{array}{l}\text { Prevalencia de } \\
\text { ejercicio físico }\end{array}$ & $\begin{array}{l}\text { N. }{ }^{\circ} \text { de } \\
\text { sujetos }\end{array}$ & $\begin{array}{l}\text { Prevalencia de } \\
\text { ejercicio físico }\end{array}$ \\
\hline \multirow{3}{*}{$\begin{array}{l}\text { Tamaño del municipio } \\
*^{* * *}\end{array}$} & $<10.001$ hab. & 896 & $80,0 \%$ & 1.324 & $91,0 \%$ \\
\hline & 10.001-50.000 hab & b. 886 & $75,9 \%$ & 1.613 & $88,2 \%$ \\
\hline & $>50.000$ hab. & 1.681 & $78,8 \%$ & 2.145 & $88,6 \%$ \\
\hline \multirow{3}{*}{$\begin{array}{l}\text { Nivel de estudios } \\
* /^{* *}\end{array}$} & De $1 .{ }^{\text {er ciclo }}$ & 1.923 & $74 ; 9 \%$ & 2.396 & $87,7 \%$ \\
\hline & De $2 .{ }^{\circ}$ ciclo & 906 & $80,7 \%$ & 1.545 & $90,2 \%$ \\
\hline & Universitarios & 634 & $86,7 \%$ & 789 & $92,5 \%$ \\
\hline \multirow{4}{*}{$\begin{array}{l}\text { Clase social } \\
* \text { ** }^{*}\end{array}$} & $|y| \mid$ & 743 & $84,0 \%$ & 1.096 & $91,4 \%$ \\
\hline & III & 834 & $80,7 \%$ & 1.256 & $89,8 \%$ \\
\hline & IV y V & 1.847 & $75,4 \%$ & 2.674 & $87,9 \%$ \\
\hline & Otros & 39 & $76,5 \%$ & 56 & $88,9 \%$ \\
\hline
\end{tabular}

*Asociación significativa 2003. * *Asociación significativa 2006.

centaje en niñas se encontraría a edades más tempranas.

La distribución de la actividad física en el tiempo libre según las variables de estado físico/mental, de estilo de vida y variables sociodemográficas se encuentra en las tablas II y III respectivamente.

Los niños cuyos padres consideran que tienen buena salud realizan más actividad física que los que tienen una mala percepción, tanto en el 2003 (79,10\% frente a $71 \%$ ) como en el 2006 (90\% frente a $81 \%$ ).

Existe una relación directamente proporcional entre el nivel de estudios de los padres y la realización de actividad física en el tiempo libre por parte de sus hijos; cuanto mayor es este nivel, mayor es el porcentaje de niños que realizan deporte, tanto en el 2003 como en el 2006.

Los resultados del análisis multivariante se muestran en las tablas IV y $\mathrm{V}$, indicando las OR junto con los intervalos de confianza de todas las categorías de las variables para ambos años (2003 y 2006).

El análisis multivariante demuestra que la edad y el sexo están asociados con la actividad física. Respecto a la edad, las probabilidades de realizar ejercicio disminuyen según se incrementan los rangos de edad, por ello el análisis muestra un riesgo de sedentarismo creciente según aumenta la edad. De hecho, en 2003, los niños con 6-7 años son 1,41 veces más activos que los de 14-15 años; y en 2006, lo son 2,12 veces más. 
Tabla IV. Análisis multivariante 2003: resultado de la asociación entre actividad física en tiempo libre y las variables de interés significativas

\begin{tabular}{|c|c|c|c|}
\hline & \multirow[b]{2}{*}{ OR } & \multicolumn{2}{|c|}{ IC 95\% } \\
\hline & & Inferior & Superio \\
\hline \multicolumn{4}{|l|}{ Edad } \\
\hline 14-15 años & 1 & & \\
\hline 12-13 años & 0,855 & 0,658 & 1,113 \\
\hline 10-11 años & 0,764 & 0,594 & 0,983 \\
\hline 8-9 años & 0,876 & 0,691 & 1,112 \\
\hline 6-7 años & 1,415 & 1,129 & 1,773 \\
\hline \multicolumn{4}{|l|}{ Sexo } \\
\hline Niña & 1 & & \\
\hline Niño & 2,131 & 1,836 & 2,474 \\
\hline \multicolumn{4}{|c|}{ Percepción de salud } \\
\hline Regular/mala & 1 & & \\
\hline Buena & 1,458 & 1,153 & 1,844 \\
\hline \multicolumn{4}{|c|}{ Nivel de estudios } \\
\hline De $1 .^{\text {er }}$ ciclo & 1 & & \\
\hline De $2 .^{\circ}$ ciclo & 1,530 & 1,175 & 1,992 \\
\hline Universitarios & 2,164 & 1,710 & 2,739 \\
\hline
\end{tabular}

En cuanto a la variable género, los datos indican que los niños son 2,13 veces más activos que las niñas en 2003 y 1,77 veces más activos en 2006.

Los niños cuyos padres consideran que sus hijos tienen una buena salud son más activos que los que tienen una mala percepción para los 2 años en estudio, siendo la asociación entre dichas variables mayor en el 2006 que en el 2003 (OR $2003=1,458$ y OR $2006=1,864$ ).

En lo referente al nivel de estudios, se observa que los niños de padres con niveles de educación universitarios, practican más actividad física que los que tienen padres con estudios inferiores. Por lo que, el riesgo de no realizar ejercicio aumenta según disminuye el nivel de estudios, tanto en 2003 como en 2006.

Otros datos resultantes únicamente del estudio de 2006 nos muestran que los niños/as que duermen un número deficiente de horas, acorde con su edad, realizan más ejercicio (1,32 veces más) que los que tienen un patrón de sueño de más horas. Cuando consideramos la variable tamaño del municipio, observamos que los niños que viven en municipios de menos de 10.000 habitantes son 1,57 veces más activos que los de municipios de más de 50.000 habitantes. 
Tabla V. Análisis multivariante 2006: resultado de la asociación entre actividad física en tiempo libre y las variables de interés significativas



Al analizar la tendencia de la variable año de la encuesta, ajustada por las variables edad y sexo, observamos un ascenso significativo de la actividad física (ORa =2,28 [IC 95\%: 2,04-2,54]) en el año 2006 respecto al año 2003. Se espera que los niños en el 2006 sean 2,28 veces más activos que los del 2003.

\section{Discusión}

El estudio mostró que, en ambos años, el porcentaje de niños activos era superior al de sedentarios. De hecho, este aumenta de un año a otro, pues en 2003 es del $78 \%$ y en 2006 del $89 \%$. Por el contrario, un artículo realizado en 2001 con una muestra de 1.358 jóvenes españoles con la misma edad, expone que menos del 30\% hacían ejercicio varias veces a la semana ${ }^{15}$. En lo que respecta a otros países, un artículo hecho en Islandia con una muestra de 3.270 niños/as entre 11 
y 16 años, concluye que el $39 \%$ eran activos en el tiempo libre; mientras que en una muestra de jóvenes de 9 a 13 años de EE. UU., el 61,5\% no participa en ninguna actividad física organizada y el $22,6 \%$ no dedica nada de tiempo libre en hacer ejercicio ${ }^{23,24}$. Por tanto, en relación a dichos países, nuestra población de estudio tiene un porcentaje considerable de niños activos.

En cuanto al sexo, los niños hacen más ejercicio que las niñas. Afianzando nuestros resultados, en un estudio de niños de 7 a 9 años de Dublín, el 53\% de los niños eran activos frente al $28 \%$ de las niñas; y en el estudio de Islandia, también se concluyó que las niñas eran más sedentarias $^{23,25}$. Esto puede ser debido a varios factores, pero principalmente a que los padres suelen incitar más a los niños al deporte desde pequeños que a las niñas, o que los juegos deportivos más populares suelen estar más asociados al tipo de juegos que les gusta a los niños $y$ no a las niñas 8 .

En tercer lugar, se observa que los niños realizan menos actividad física a medida que se hacen mayores. De nuevo, se vuelve a coincidir con las conclusiones del estudio de Islandia, donde la prevalencia de la actividad disminuye con la edad ${ }^{23}$.

También se demostró una relación significativa entre la realización de ejercicio con la valoración de los padres sobre el estado de salud de su hijo/a. Se confirma que los niños más sedentarios son aquellos cuyos padres consideran que tienen mala salud. Ante esto, es preciso tener en cuenta que algunos de estos niños presentan alguna enfermedad, como pueden ser asma o alergias, que influyen en la realización de ejercicio. De hecho, un estudio de casos y controles hecho en EE. UU. en 2004, demuestra que los niños con asma son más sedentarios que los niños sanos ${ }^{26}$.

Por otra parte, el nivel de estudios de los padres tiene influencia sobre el ejercicio del menor; cuanto mayor es este nivel, mayor es el porcentaje de niños que realizan deporte, tanto en 2003 como en 2006. Un estudio de EE. UU. indica que los niveles más bajos de educación parental, están asociados con un mayor declive de la actividad física en niñas blan$\mathrm{Cas}^{27}$. Estos resultados difieren del estudio de M. H. Oehlschlaeger et al. de 1996, en el que se indica que cuanto mayor es la educación de la madre, mayor es el sedentarismo del hijo/a $\mathrm{a}^{14,16}$.

En el análisis univariante, se encontró asociación entre el tipo de dieta de los niños y actividad física, pero en el multivariante, la magnitud estimada cambia ligeramente y el nivel de significación fue nulo. Así, no podemos mostrar que exis- 
ta una asociación entre el tipo de alimentación y actividad física. Ocurre lo mismo con la clase social y el tamaño del municipio, para ambos años.

Hay una serie de limitaciones en este estudio. Primero, el uso de encuestas como la que hemos utilizado (ENS) pueden suponer una limitación o sesgo, ya que utilizan datos indirectos proporcionados por los padres de los niños en estudio. En segundo lugar, la clasificación de la variable dependiente, cómo realiza o no realiza actividad física, sin tener en cuenta la frecuencia, la intensidad y el tiempo dedicado a la actividad física. En tercer lugar, cualquier información obtenida mediante una entrevista puede estar sujeta al sesgo de memoria (en este caso de los padres) o al sesgo del entrevistador. Finalmente, la tasa de respuesta de la ENS no es del $100 \%$, encontrándose un elevado porcentaje de falta de respuesta en el cuestionario de evaluación respecto a ciertas variables de interés, lo cual supone también una limitación para el estudio. A pesar de todo esto, se cumple con los requisitos de la Organización Mundial de Salud para la ENS ${ }^{15}$.

Pese a estas limitaciones, creemos que los resultados muestran la distribución de la población joven española en relación con la práctica de actividad física en el tiempo libre y revelan relaciones potencialmente importantes entre actividad física y otros factores. Este análisis puede tener un doble uso: en primer lugar, los datos resultantes pueden servir de guía para el desarrollo de nuevas estrategias y medidas de promoción del ejercicio físico en este grupo de población. En segundo lugar, los resultados pueden ser fundamento para futuras investigaciones, con el fin de ayudar y mejorar las recomendaciones sobre la actividad física ${ }^{15}$.

La actividad física viste gran importancia en la niñez y en la juventud, y debería seguir ejercitándose en la edad adulta ${ }^{12}$. Hay estudios que resaltan la importancia de desarrollar hábitos y destrezas de actividad física durante la niñez como vía para aumentar la probabilidad de adquirir un estilo de vida activo a edades tardías. Por tanto, deberían de llevarse a cabo programas de promoción de actividad física enfocados a población joven.

En España, el Ministerio de Sanidad y Consumo ha puesto en marcha en el 2005, la Estrategia para la Nutrición, Actividad Física y Prevención de la Obesidad (NAOS), que tiene como finalidad mejorar los hábitos alimentarios e impulsar la práctica regular de la actividad física de todos los ciudadanos, poniendo especial atención en la prevención durante la etapa infantil ${ }^{10}$. 
Esperamos que este estudio sirva de impulso y ayuda para poner en marcha más estrategias de promoción de la actividad física.

\section{Conclusiones}

A medida que los niños/as se acercan a la pubertad se hacen más sedentarios, es decir, practican menos ejercicio físico. Respecto al sexo, los niños hacen más ejercicio que las niñas durante todos los años de su juventud. Por otro lado, los niños menos activos son aquellos cuyos padres consideran que tienen mala salud. Y los niños de padres con niveles de educación universitarios, practican más actividad física que los que tienen padres con estudios inferiores.

La sociedad española es cada vez más consciente de los beneficios que aporta la práctica de actividad física, de ahí que, con el paso de los años, observemos un ascenso significativo de la actividad física.

\section{Bibliografía}

1. Mur de FL, Fleta ZI, Garagorri Otero JM, Moreno $A L$, Bueno SM. Physical activity and leisure time in children. II: Relationship with dietary habits. An Esp Pediatr. 1997;46:126-32.

2. Pastor $Y$, Balaguer I, Pons D, García-Merita $M$. Testing direct and indirect effects of sports participation on perceived health in Spanish adolescents between 15 and 18 years of age. J Adolesc. 2003;26:717-30.

3. Dietz WH. Health consequences of obesity in youth: childhood predictors of adult disease. Pediatrics. 1998;101:518-25.

4. Slattery ML, Edwards SL, Ma KN, Friedman GD, Potter JD. Physical activity and colon cancer: a public health perspective. Ann Epidemiol. 1997;7: 137-45.

5. Wang G, Dietz WH. Economic burden of obesity in youths aged 6 to 17 years: 1979-1999. Pediatrics. 2002;109:E81.

6. Bidoli E, Schinella D, Franceschi S. Physical ac- tivity and bone mineral density in Italian middleaged women. Eur J Epidemiol. 1998;14:153-7.

7. Van Der HK, Paw MJ, Twisk JW, Van MW. A brief review on correlates of physical activity and sedentariness in youth. Med Sci Sports Exerc. 2007;39:1241-50.

8. Merino Merino B, González E, Aznar Laín S, Castro Ulled JM, Veiga Núñez O. Guía para padres y madres. Madrid: Ministerio de Sanidad y Consumo; 2008 [consultado el 01/03/2009]. Disponible en www.msc.es/ciudadanos/proteccionSalud/ado lescencia/actividad_fisica.htm

9. Ara I, Vicente-Rodríguez $G$, Jiménez-Ramírez J, Dorado C, Serrano-Sánchez JA, Calbet JA. Regular participation in sports is associated with enhanced physical fitness and lower fat mass in prepubertal boys. Int J Obes Relat Metab Disord. 2004; 28:1585-93.

10. Prevención de la obesidad infantil. Madrid: Ministerio de Sanidad y Consumo; 2006 [consultado el 01/03/2009]. Disponible en www.msc.es/ campannas/campanas06/obesidadlnfant3.htm 
11. Kemper HC, Post GB, Twisk JW, Van MW. Lifestyle and obesity in adolescence and young adulthood: results from the Amsterdam Growth and Health Longitudinal Study (AGAHLS). Int J Obes Relat Metab Disord. 1999;23 Suppl 3:s34s40.

12. Montil M, Aznar S, Barriopedro M. Cumplimiento de las recomendaciones de actividad física en una muestra de niños de la Comunidad Autónoma de Madrid. Universidad de Extramadura; 1998 [consultado el 01/03/2009]. Disponible en www.unex.es/eweb/cienciadeporte/congreso/ 04\%20val/pdf/c24.pdf

13. Mo F, Turner M, Krewski D, Mo FD. Physical inactivity and socioeconomic status in Canadian adolescents. Int J Adolesc Med Health. 2005;17: 49-56.

14. Gordon-Larsen P, McMurray RG, Popkin BM. Determinants of adolescent physical activity and inactivity patterns. Pediatrics. 2000;105:E83.

15. Lasheras L, Aznar S, Merino B, López EG. Factors associated with physical activity among Spanish youth through the National Health Survey. Prev Med. 2001;32:455-64.

16. Oehlschlaeger $M H$, Pinheiro RT, Horta $B$, Gelatti C, San'Tana P. Prevalence of sedentarism and its associated factors among urban adolescents. Rev Saude Publica. 2004;38:157-63.

17. Pastor Y, Balaguer I, García-Merita M. The relationship between self-concept and a healthy lifestyle in adolescence: an exploratory model. Psicothema. 2006;18:18-24.

18. Instituto Nacional de Salud. Encuesta Nacional de Salud 2003: Metodología detallada. Madrid: Instituto Nacional de Estadística; 2008 [consultado el 01/03/2009]. Disponible en www.ine. es/metodologia/t15/t1530419.pdf

19. Instituto Nacional de Salud. Encuesta Nacional de Salud 2003: Evaluación de la falta de respuesta. Madrid: Instituto Nacional de Estadística;
2008 [consultado el 01/03/2009]. Disponible en www.msc.es/estadEstudios/estadisticas/docs/eval frsalud_definitivo.pdf

20. Instituto Nacional de Salud. Encuesta Nacional de Salud 2006: Metodología detallada. Madrid: Instituto Nacional de Estadística; 2008 [consultado el 01/03/2009]. Disponible en www.msc. es/estadEstudios/estadisticas/encuestaNacional/ encuestaNac2006/metodENS2006.pdf

21. Instituto Nacional de Salud. Encuesta Nacional de Salud 2006: Evaluación de la falta de respuesta. Madrid: Instituto Nacional de Estadística; 2008 [consultado el 01/03/2009]. Disponible en www.msc.es/estadEstudios/estadisticas/encuesta Nacional/encuestaNac2006/evaluacionFalta Resp.pdf

22. Vázquez Martínez C, Alcaraz Cebrián F, Garriga García M, Martín E, Montagna MC, Ruperto MM, y cols. Dietética y nutrición: grupos de alimentos. Fisterra. Atención Primaria en la Red [Internet] [consultado el 01/03/2009]. Disponible en www. fisterra.com/material/Dietetica/GruposAli mentos.asp [actualizado el 14/11/2007].

23. Kristjansdottir G, Vilhjalmsson R. Sociodemographic differences in patterns of sedentary and physically active behavior in older children and adolescents. Acta Paediatr. 2001;90:429-35.

24. Physical activity levels among children aged 9-13 years, United States, 2002 MMWR. Morb Mortal Wkly Rep. 2003;52:785-8.

25. Hussey J, Gormley J, Bell C. Physical activity in Dublin children aged 7-9 years. Br J Sports Med. 2001;35:268-72.

26. Lang DM, Butz AM, Duggan AK, Serwint JR. Physical activity in urban school-aged children with asthma. Pediatrics. 2004;113:e341-e6.

27. Kimm SY, Glynn NW, Kriska AM, Barton BA, Kronsberg SS, Daniels SR, et al. Decline in physical activity in black girls and white girls during adolescence. N Engl J Med. 2002;347:709-15. 